\title{
Enterocutaneous fistulas: a primer for radiologists with emphasis on CT and MRI
}

\author{
Massimo Tonolini $^{1} \cdot$ Paolo Magistrelli ${ }^{1}$
}

Received: 29 June 2017 /Revised: 22 August 2017 / Accepted: 24 August 2017 / Published online: 29 September 2017

(C) The Author(s) 2017. This article is an open access publication

\begin{abstract}
Enterocutaneous fistulas (ECFs) represent abnormal communications between the gastrointestinal tract and the skin. Nowadays, the majority ( $\sim 80 \%)$ of ECFs develops secondary to abdominal surgeries; alternative, less common causes include chronic inflammatory bowel diseases (IBD) such as Crohn's disease, tumours, and radiation enteritis in descending order of frequency. These rare disorders require thorough patient assessment and multidisciplinary management to limit the associated morbidity and mortality. This pictorial review includes an overview of causes, clinical manifestations, complications and management of ECFs. Afterwards, the imaging appearances, differential diagnoses, and therapeutic options of post-surgical, IBD-related, and malignant ECFs are presented with case examples. Most of the emphasis is placed on the current pivotal role of $\mathrm{CT}$ and MRI, which comprehensively depict ECFs providing cross-sectional information on the underlying postsurgical, neoplastic, infectious, or inflammatory conditions. Radiographic fistulography remains a valid technique, which rapidly depicts the ECF anatomy and confirms communication with the bowel. The aim of this paper is to increase radiologists' familiarity with ECF imaging, thus allowing an appropriate choice between medical, interventional, or surgical treatment, ultimately resulting in higher likelihood of therapeutic success.
\end{abstract}

Massimo Tonolini

mtonolini@sirm.org

1 Department of Radiology, "Luigi Sacco" University Hospital, Via G.B. Grassi 74, 20157 Milan, Italy
Teaching Points

- Enterocutaneous fistulas may complicate abdominal surgery, sometimes Crohn's disease and tumours.

- The high associated morbidity and mortality result from sepsis, malnutrition and metabolic imbalance.

- The multidisciplinary management of ECFs requires thorough imaging for correct therapeutic choice.

- Radiographic fistulography rapidly depicts fistulas and communicating bowel loops in real-time.

- Multidetector CT and MRI provide cross-sectional information on fistulas and underlying diseases.

Keywords Enterocutaneous fistula $\cdot$ Malignant fistula $\cdot$ Crohn's disease $\cdot$ Computed tomography $(\mathrm{CT}) \cdot$ Magnetic resonance imaging (MRI)

\section{Introduction}

Enterocutaneous fistulas (ECFs) are defined as abnormal communications between the gastrointestinal (GI) tract and the skin. Albeit relatively rare compared to past decades, ECFs still represent one of the most challenging conditions encountered in surgical practice, burdened with severe morbidity, impaired quality of life, and substantial mortality [1-3].

In recent years, specialised centres developed a robust management approach, which combines experience from surgeons, interventional radiologists, intensive care physicians, nutritionists, wound care specialists and nurses. Summarised in Table 1, this multidisciplinary treatment relies on thorough diagnostic imaging for correct patient selection and therapeutic choice: unfortunately, very limited literature exists on cross-sectional imaging of ECFs $[4,5]$.

This pictorial review relies upon experience at a tertiary referral hospital where oncologic and chronic inflammatory 
Table 1 Fundamentals of the current multispecialist approach to enterocutaneous fistula management

\begin{tabular}{|c|c|c|}
\hline Step no. & Treatment basics & Comments \\
\hline 1 & Control of sepsis & $\begin{array}{l}\text { Often requiring intensive care support } \\
\text { Imaging-guided drainage of abscess collections }\end{array}$ \\
\hline 2 & Limiting output volume & $\begin{array}{l}\text { Octreotide administration } \\
\text { Bowel rest, enteral, or parenteral nutrition }\end{array}$ \\
\hline 3 & Wound or skin care & $\begin{array}{l}\text { Use of dressings or bags depending on output } \\
\text { Suction or Vacuum-Assisted Closure (VAC) devices if available }\end{array}$ \\
\hline 4 & $\begin{array}{l}\text { Metabolic and nutritional } \\
\text { optimisation }\end{array}$ & $\begin{array}{l}\text { Adequate hydration, electrolytes balance, nutritional status } \\
\text { Proximal versus location in the bowel influences nutritional } \\
\text { and fluid requirements }\end{array}$ \\
\hline 5 & $\begin{array}{l}\text { Assessing likelihood of } \\
\text { spontaneous closure }\end{array}$ & $\begin{array}{l}\text { Versus percutaneous treatment or elective surgical repair } \\
\text { Factors associated with favourable healing: } \\
\text { - narrow-calibre and/or relatively long }(>2 \mathrm{~cm}) \mathrm{ECFs} \text {, } \\
\text { - small enteric defect or anastomotic dehiscence }(<1 \mathrm{~cm}) \\
\text { Factors associated with probable non-healing: } \\
\text { - presence of foreign bodies, } \\
\text { - history of irradiation, } \\
\text { - active infection, } \\
\text { - untreated chronic inflammatory bowel disease, } \\
\text { - untreated tumours, } \\
\text { - distal obstruction }\end{array}$ \\
\hline
\end{tabular}

bowel disease (IBD) surgeries are performed. It includes an overview of causes, clinical manifestations, complications, and management of ECFs. Afterwards, the imaging appearances, differential diagnoses and therapeutic options of postsurgical, chronic inflammatory bowel diseases (IBD)-related and malignant ECFs are presented with examples. Most of the emphasis is placed on the current pivotal role of CT and MRI, which comprehensively depict ECFs providing crosssectional information on the underlying postsurgical, neoplastic, infectious, or inflammatory disorders.

Our aim is to increase radiologists' familiarity with ECF imaging, thus allowing an appropriate choice between medical, interventional, or surgical treatment, ultimately resulting in higher likelihood of therapeutic success.

\section{Clinical overview of enterocutaneous fistulas}

The origin of an ECF may lie anywhere along the GI tract, and is found in the small bowel, colon, stomach, and duodenum in descending order of frequency. Nowadays, the majority (75$85 \%$ ) of ECFs develop postoperatively following oncologic abdominal surgery, bowel resections for Crohn's disease (CD), ventral hernioplasty, and repeated laparotomies. The remaining, spontaneous ECFs occur secondary to IBD (mostly $\mathrm{CD}$ rather than indeterminate and ulcerative colitis), fistulising tumours, radiation enteritis, colonic diverticulitis, intra-abdominal sepsis, and trauma, in descending order of frequency $[1-3,6]$.

The hallmark clinical finding is the external fistulous opening (EFO) at the anterior or lateral abdominal wall, which is often surrounded by an inflamed, tender cutaneous region, and may sometimes be hidden beneath a skin fold, or lie in the site of a recent or still patent surgical incision. Discharge may be either intermittent or continuous, sometimes purulent or faecal. The ECF output may be quantified and categorised as low (below $200 \mathrm{~mL} /$ day), moderate or high (over $500 \mathrm{~mL} /$ day). The macroscopic and biochemical features of the effluent provide useful clues to the ECF origin [1-3, 6].

Historically, the ECF-related mortality was in excess of $50 \%$, particularly in those patients with high-output fistulas, tumours and sepsis. During the last 15 years, the improvements in surgery, wound care, nutritional and metabolic support resulted in decreased fatality rates $(8-13 \%)$ and ultimate therapeutic success after a median 6 weeks in $75-91 \%$ of patients $[1-3,6]$.

However, rates of nonoperative healing remain low ( $\sim 20-27 \%$ of cases overall) and ECFs still represent chronic, debilitating conditions associated with prolonged intensive care unit and hospital stays. The ECF-associated morbidity arises from sepsis, malnutrition, and metabolic imbalance. The invariable fluid and electrolyte abnormalities result from the combined effect of protracted catabolic febrile state, ileus or obstruction, loss of bowel integrity, and absorption. Further complications include generalised peritonitis, abdominal wall 
abscesses, and necrotising fasciitis secondary to soft tissue damage by bacteria and digestive juices [7, 8].

The ECF aetiology is the single best prognostic factor and predictor of therapeutic success [9]. In fact, the cause and underlying abnormalities strongly influence the likelihood of spontaneous closure and dictate the need for additional medical, surgical, or interventional treatment; therefore, the following sections will present the postoperative, CD-related and malignant ECFs separately with their specific imaging features and therapeutic approaches $[1-3,6]$.

\section{Post-surgical enterocutaneous fistulas}

\section{Clinical features}

Despite improved techniques, most ECFs currently develop after surgical procedures, secondary to anastomotic leakage, disruption of repaired enterotomy, or inadvertent intraoperative bowel injury. Some series reported an alarming incidence $(0.8-2 \%)$ of postsurgical ECFs, probably resulting from the increasing complexity of procedures such as cytoreductive surgery with peritonectomy. Currently, the majority of ECFs are encountered in patients operated for colorectal and ovarian cancers. Alternatively, ECFs may develop following nononcologic surgery such as ventral hernioplasty using prosthetic mesh, or repeated laparotomies such as for adhesiolysis and recurrent bowel obstruction. In our experience, the majority of patients had complex histories including multiple surgical interventions [4, 10-12].

Post-surgical ECFs may manifest early (within the first postoperative week) with a combination of septic fever, tachycardia, and hypotension, respiratory distress, progressively distended bowel, localised or diffuse abdominal tenderness, acidosis, leukocytosis, and elevated C-reactive protein. Alternatively, ECFs may present late during prolonged postoperative hospitalisation or after discharge as a tender, erythematous, fluctuant area at the surgical incision, or laparoscopic port site; in such cases nurses often notice medication gauzes becoming wet with either bile, enteric material, or stool [2].

\section{Radiographic fistulography: technique and appearances}

Fistulography (sinography) traditionally represented the mainstay technique to investigate ECFs and currently remains useful as a complementary technique to confirm communication between the EFO and a segment of the gastrointestinal tract. In our experience, this technique is best performed with help from the referring surgeon, who probes the EFO and enters the ECF. Depending on its calibre, a venous cannula or angiographic needle, a standard syringe, a Foley-type catheter, a paediatric or nasogastric feeding tube may be used. Then, non-forceful manual injection of low-osmolar water-soluble iodinated contrast medium (CM) is performed under fluoroscopic observation. Inexpensive, rapid, and easy to perform, fistulography directly visualises the communicating small bowel (Fig. 1A, B) or colonic segment (Fig. 1C) and provides information on ECF anatomy such as calibre, length, course, and ramifications. False negative studies may result from oedema, debris, or compression by abscess or mass impeding $\mathrm{CM}$ flow into the intestinal lumen. However, fistulography lacks cross-sectional visualisation of mural and extraluminal pathology upstream, downstream, and at site of the ECF [4, 5].

\section{CT techniques, appearances, and differential diagnoses}

Nowadays the vast majority of patients should undergo CT or MRI studies to provide comprehensive cross-sectional information on ECFs and underlying disorders as a consistent basis for appropriate treatment choice and planning. Multidetector $\mathrm{CT}$ is preferred in uncooperative or severely ill patients such as those in early postoperative hospitalisation, because it obtains high-spatial resolution images in seconds, thus limiting motion or peristalsis artefacts. In the setting of ECF, intravenous CM is warranted unless contraindicated. Orally administered CM opacification may be helpful in patients with scarce intra-abdominal fat, to ease differentiation of bowel loops from extraluminal structures and abnormal collections; unfortunately, most patients who recently underwent surgery have ileus or obstruction and are unable to drink diluted CM. Alternatively, CT-fistulography (Fig. 2) with prior injection of iodinated $\mathrm{CM}$ such as $3 \%$ diluted iopamidol or iohexol through the EFO represents a useful one-stop-shop technique, which combines cross-sectional information with opacification of involved bowel tract. Image reconstruction and study interpretation along the sagittal plane are strongly recommended, as it provides the best visualisation of the ventral abdominal wall $[4,5]$.

The usual CT appearance of an ECF (Fig. 3) includes a tubular structure originating from a bowel loop, which directs ventrally or ventro-laterally through the peritoneum, crosses the abdominal wall muscles and fasciae to reach the cutaneous surface. ECFs may appear either collapsed or patent with gaseous and/or fluid content, in the latter case resulting in the characteristic "tram-track" appearance. Fistulous walls may be more or less thick, sometimes measuring up to one centimetre (Fig. 3C, D). The formation of a peripherally enhancing abscess with fluid and gaseous content along the ECF track (Fig. 4) should be reported, since it generally modifies the therapeutic approach.

In our experience, postsurgical ECFs often develop in cachectic patients with very thin subcutaneous and muscular planes of the ventral abdomen following repeated surgeries. The involved bowel loops tend to converge towards the ECF site and adhere closely to the peritoneal serosa (Figs. 2 and 5); 


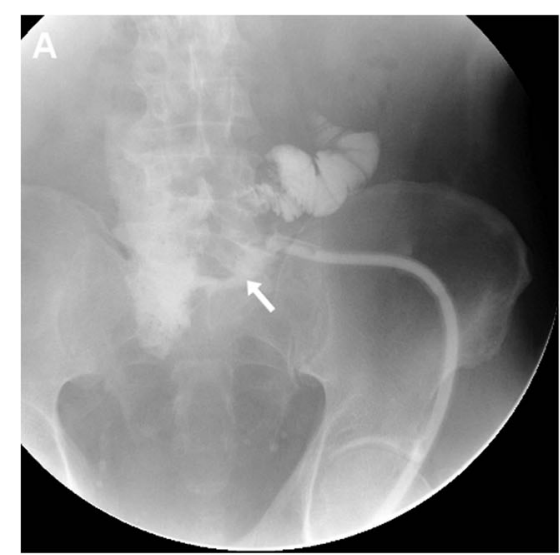

Fig. 1 Two fistulography examples. A Fistulography performed via a catheter piercing through the external fistulous opening (EFO) in a 74year-old man with history of repeated surgeries to manage bowel obstruction, adhesions and anastomotic dehiscence: optimal visualisation of course and length of the enterocutaneous fistula

(ECF, arrow), in

not unusually, an ECF develops within a post-incisional hernia (Fig.3C, D).

At CT, the EFO generally corresponds to a focal cutaneous retraction or depression, and is often surrounded by thickened inflamed skin (Figs. 3 and 4); alternatively, a frank cutaneous breach or discontinuity may be observed, particularly in highoutput ECFs (Fig. 5).

The two key CT differential diagnoses of an ECF are represented by:

- A recent laparotomic incision (Fig. 3A), which is differentiated by reviewing the surgical description and inspecting the patient's abdomen, and

- Those fistulas (with usual "tram-track" CT appearance), which drain externally an intra-abdominal postsurgical collection (Fig. 6A, B), but lack communication with the bowel.

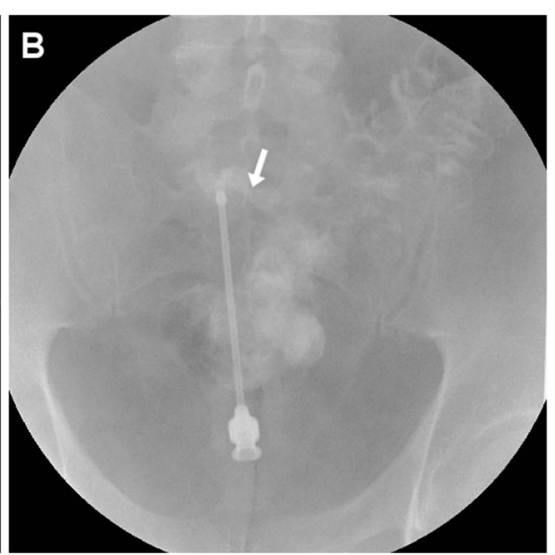

communication with the ileum. B Fistulography using a needle in a 60year-old HIV-positive man with recent acute colonic diverticulitis: the ECF (arrow) communicating with the sigmoid colon required left hemicolectomy and ECF debridement

\section{MRI technique and findings}

Borrowing from extensive experience with perianal fistulas, MRI has intrinsically superior soft tissue contrast compared to $\mathrm{CT}$ and may, therefore, better define the fistulous tract; furthermore, MRI is appealing to both radiologists and patients because of absent ionising radiation exposure. However, in critically ill patients such as those in the early postoperative days, MRI is hampered by respiratory and peristaltic artefacts from bowel obstruction or ascites. In patients who can sufficiently cooperate, MRI protocols to investigate ECFs should rely on multiplanar fluid-sensitive T2-weighted images including the anterior abdominal wall; the use of fat suppression techniques in at least one plane is recommended to improve detection of oedema and inflammation in the intra-abdominal and subcutaneous fat. Additionally, intravenous administration of gadolinium $\mathrm{CM}$ is helpful to visualise hypervascularity
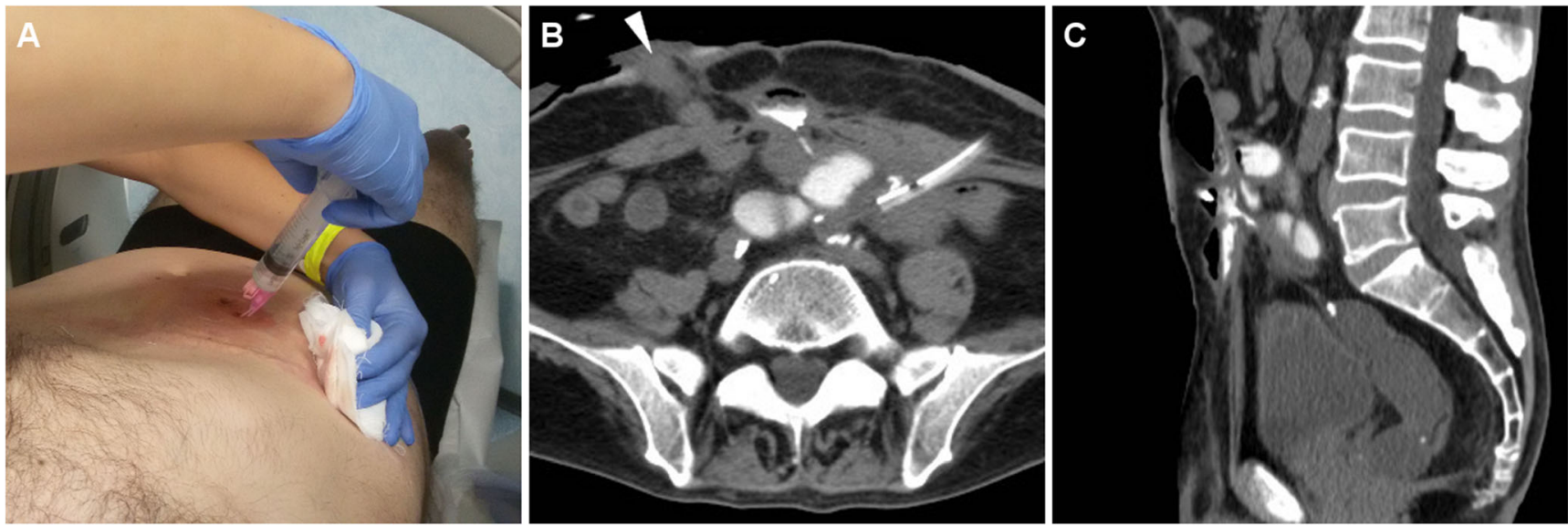

Fig. 2 CT-fistulography technique, directly performed on the scanner table by manual injection through the EFO of diluted CM using a syringe and a venous cannula (A). An example of CT-fistulography $(\mathbf{B}, \mathbf{C})$ in a 69 -year-old man with ulcerative colitis, previous right hemicolectomy and repeated surgery for complications: shortly after ileostomy closure (note drainage tube) low-output ECF was diagnosed, with communication between the EFO (arrowhead in B) and the opacified ileum 
Fig. 3 Early postoperative ECF in a 74-year-old woman following cytoreductive surgery for advanced endometrial carcinoma with peritoneal carcinomatosis. Characteristic CT $(\mathbf{A}, \mathbf{B})$ findings included enhancing "tram-track" paired structures (arrows) crossing from the peritoneal cavity through the anterior abdominal wall to the EFO (arrowheads), separated from the recent laparotomic access site (+). The ECF ultimately healed with prolonged in-hospital conservative treatment

Another ECF developing in a 54year-old obese woman with postincisional abdominal evisceration: corresponding CT $(\mathbf{C}, \mathbf{D})$ appearance included thick-walled enhancing fistula (arrows) coursing from a herniated small bowel loop to the focally retracted $\mathrm{EFO}$ (arrowheads). Surgical treatment included ECF debridement and mesh hernioplasty
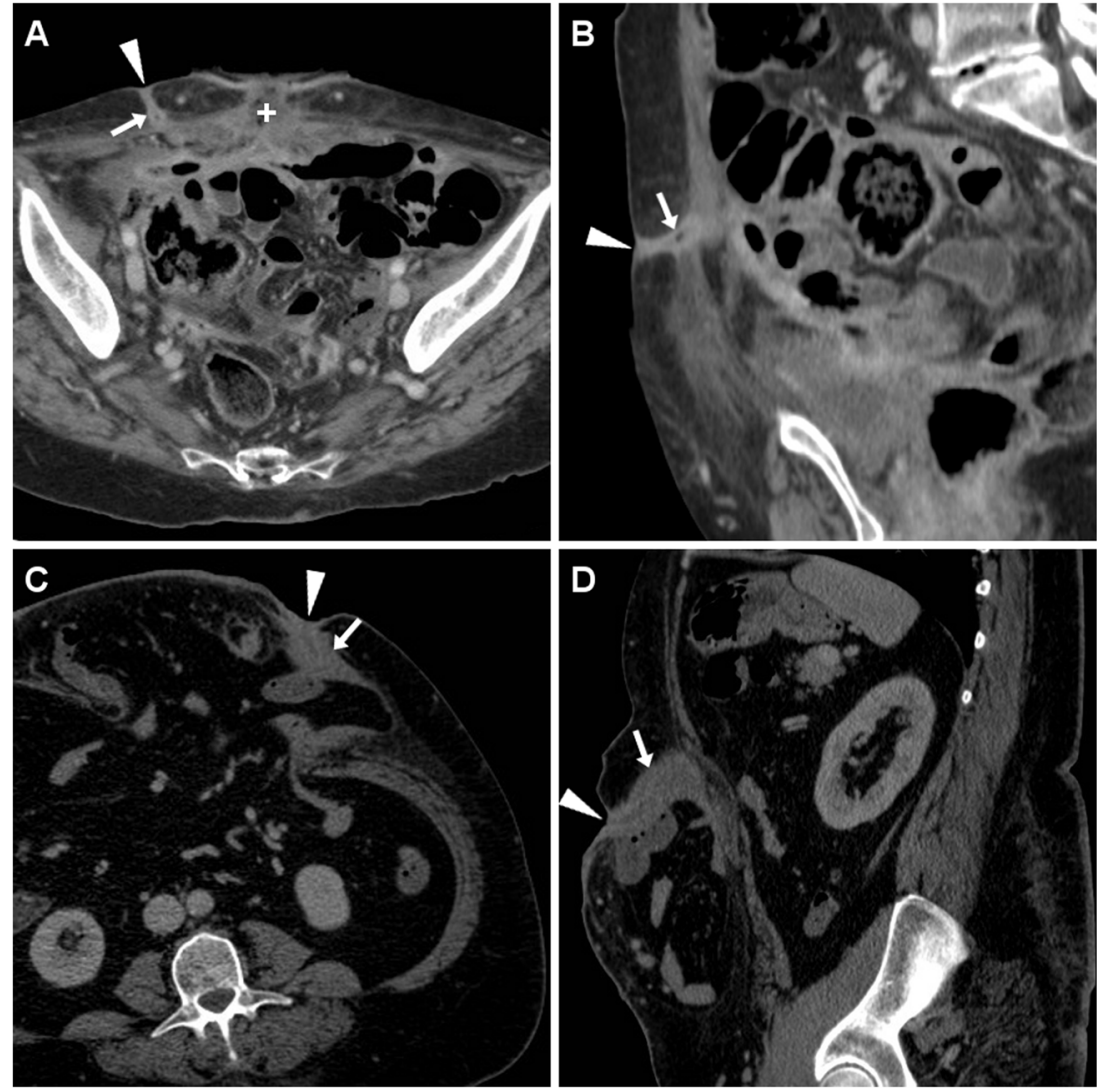

of ECF walls, abscess collections and surrounding fat planes, which is best appreciated on volumetric fat-suppressed T1weighted gradient-echo sequences such as T1 highresolution isotropic volume excitation (THRIVE), liver acquisition with volume acquisition (LAVA) or volumetric interpolated breath-hold examination (VIBE).

On heavily T2-weighted MRI sequences, the ECF is seen as a fluid-filled hyperintense tubular structure coursing through the abdominal wall and subcutaneous fat to reach the EFO. The fistulous walls show variable, generally intermediate signal intensity on both T1- and T2-weighted acquisitions, with corresponding "tram-track" enhancement (Fig. 7) on post-gadolinium images. Over time, persistent ECFs tend to develop thicker walls with lower T2-weighted signal from fibrosis, and decreasing intensity of contrast enhancement. Unfortunately, in our experience identification of the

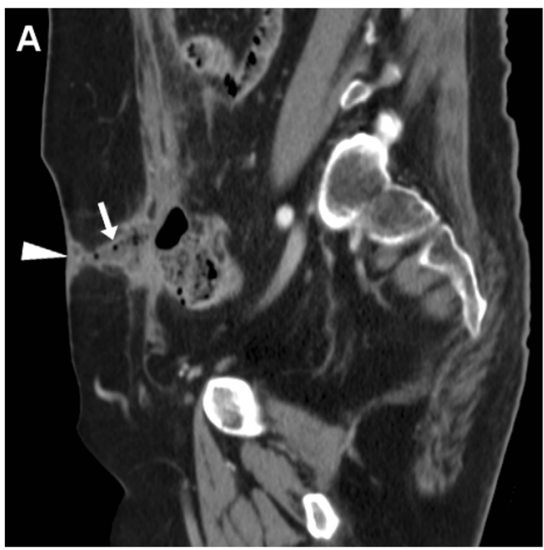

Fig. 4 Postoperative ECF with subcutaneous abscess formation in a 71-year-old woman, following resection of sigmoid colon diverticulitis, complicated by anastomotic dehiscence which required re-laparotomy, ileal resection, and temporary ileostomy. CT diagnosed communication

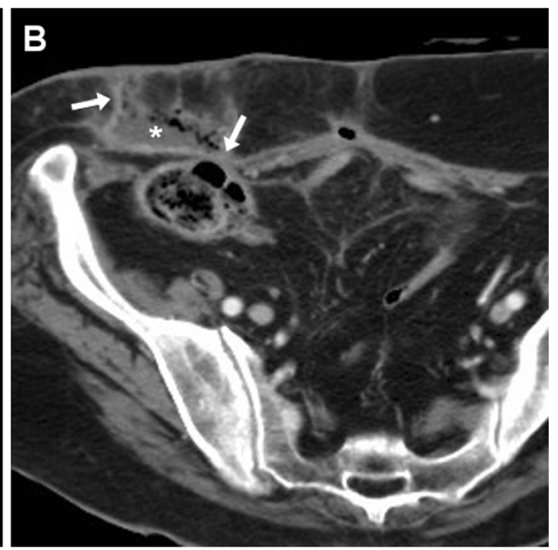

(arrows) between the cecum and an abscess $(*)$ located just superficially to the ventral abdominal muscles, draining to the EFO (arrowhead in $\mathbf{A})$. Surgical treatment included ileocecal resection and abscess toilette 


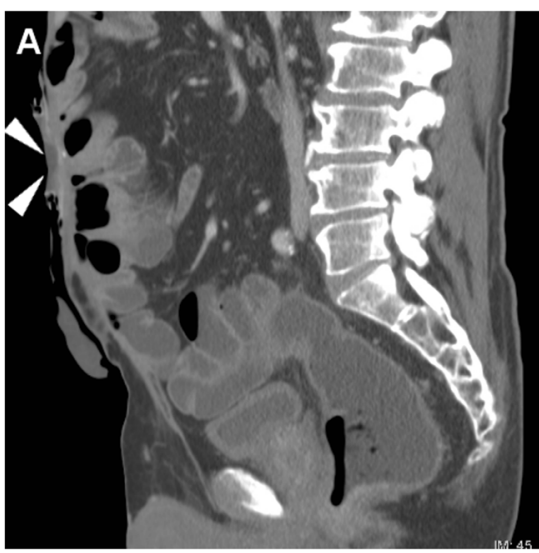

Fig. 5 High-output ECF in a 69-year-old man with history of repeated surgeries to manage jejunal and duodenal perforations from ZollingerEllison syndrome, followed by hemicolectomy, ileostomy, and colostomy because of ischemic bowel necrosis. At $\mathrm{CT}$, the wide draining $\mathrm{EFO}$

presence or absence of bowel communication is generally more challenging at MRI (Fig. 6C, D) compared to CT [4, 5].

\section{Treatment}

Currently, the majority of postoperative ECFs tend to heal with conservative measures, endoscopic or percutaneous therapies. The latter consist in local injection of sealants such as cyanoacrylic glue (Glubran 2, GEM, Viareggio, Italy), and is rapidly becoming the preferred option as it achieves therapeutic success in a single session in almost two-thirds of cases, without any complications $[5,13]$.

Endoscopic positioning of clips and stents is the mainstay option when a leaking bowel anastomosis is present. Surgical repair is nowadays reserved for selected cases and remains associated with significant morbidity; high expertise is required to perform adhesiolysis and bowel resection at the

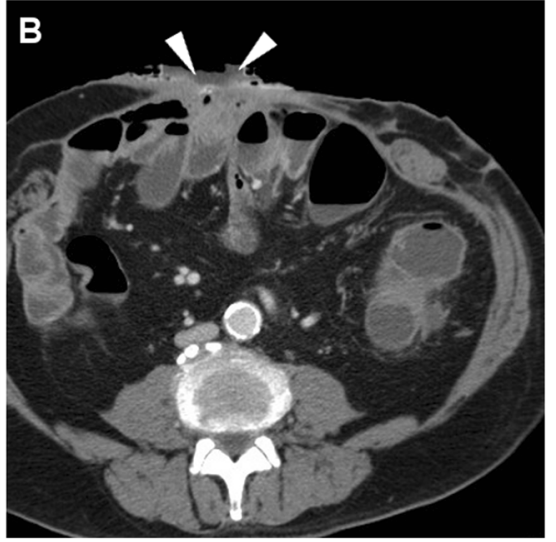

corresponded to a 2-cm hypoattenuating discontinuity (arrowheads) in the thickened skin, closely adherent to distended bowel loops with airfluid levels consistent with postoperative ileus

internal fistula opening; unfortunately, postoperative ECF recurrence is not uncommon (16-21\% of cases) [3, 14].

\section{Enterocutaneous fistulas in chronic inflammatory bowel diseases}

\section{Clinical features}

In patients with IBD, enterocutaneous fistulas may be either spontaneous or postoperative. The former situation results from the characteristic transmural inflammation of the affected enteric wall, which leads to penetration into the adjacent tissues, sometimes involves the abdominal wall and may ultimately reach the superficial tissues. In the vast majority of cases, ECFs arise from the diseased distal ileum and are associated with long-standing or active
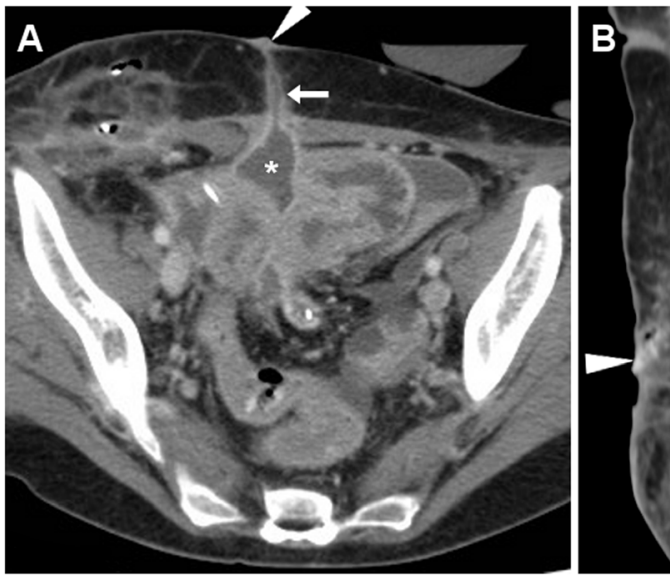

Fig. 6 Two examples of postsurgical fistulas draining intra-abdominal collections, without bowel involvement. In a 34-year-old woman, after ileocecal resection CT (A, B) showed an EFO (arrowheads) communicating via a "tram-track" fistula (arrows) through the abdominal wall with

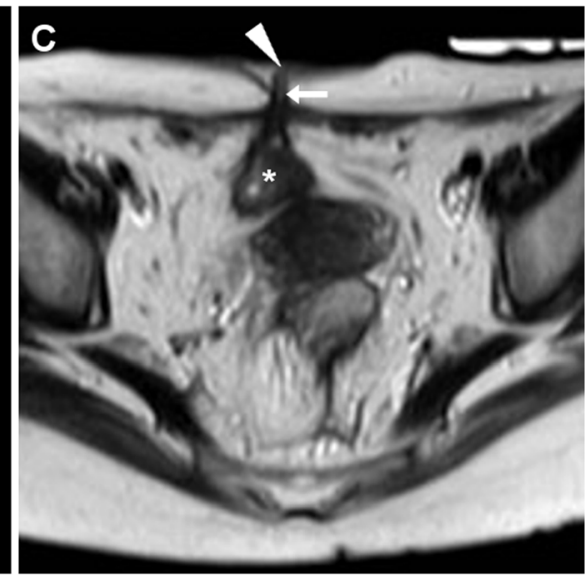

a peripherally enhancing collection $(*)$ which ultimately resolved. In a 48 year-old woman with recent CD resection, T2-weighted MRI (C) depicted a chronic postsurgical collection $(*)$ connected to the skin by a fistula (arrows) 

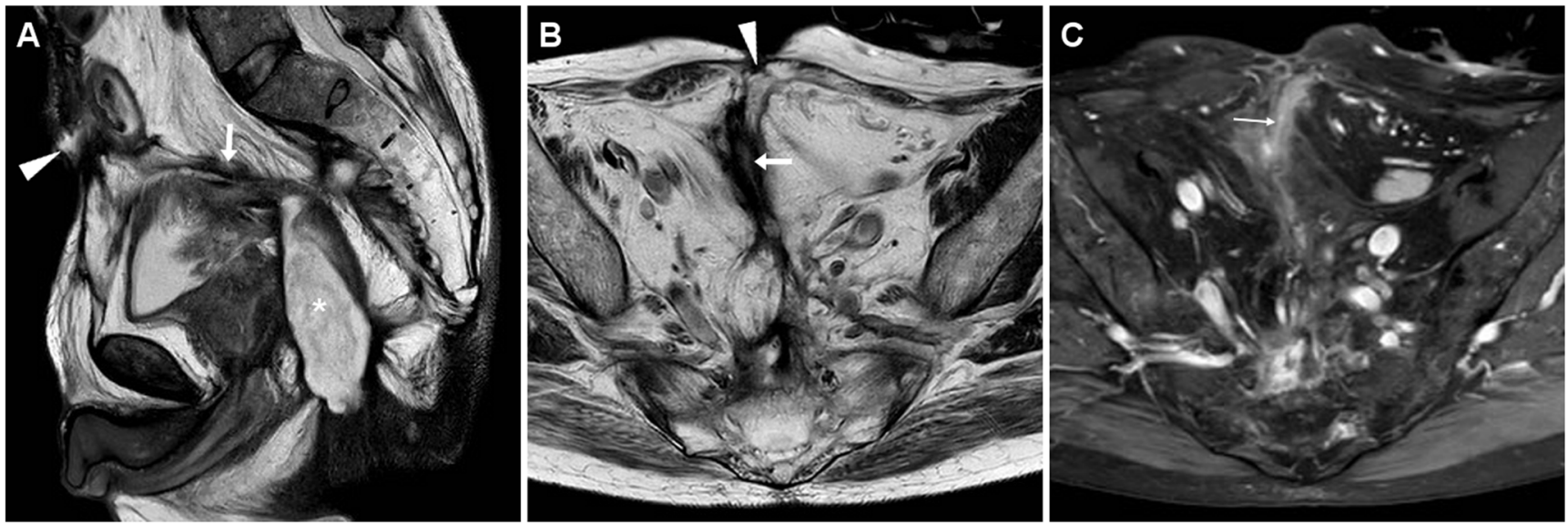

Fig. 7 MRI diagnosis of post-surgical ECF in a 65-year-old male with long-standing fistulising $\mathrm{CD}$, previous abdomino-perineal resection for perianal fistulas complicated by anal squamocellular carcinoma (ASC). On T2-weighted images, the fluid-filled ECF (arrows) was seen coursing ventrally to reach the ventral EFO (arrowheads) at the site of past surgical

fistulising CD; exceptionally, an ECF may be the initial manifestation of an undiagnosed IBD. Albeit uncommon, CD still represents the main cause of non-iatrogenic ECFs and should therefore be suspected in a non-healing spontaneous fistula in a young or middle-aged, otherwise healthy patient $[15,16]$.

\section{Cross-sectional imaging}

According to the European Crohn's and Colitis Organisation (ECCO) statements, CT- and MR-enterography with oral luminal distension represent the ideal techniques for imaging diagnosis and follow-up of $\mathrm{CD}$, with similar indications and diagnostic accuracy; the latter modality is increasingly used since it does not use ionising radiation. In selected patients, water-enema multidetector CT may be useful to visualise the extent, mural and extraluminal changes of colonic IBD. In urgent conditions, standard contrast-enhanced $\mathrm{CT}$ is widely used and highly helpful when acute CD complications such as perforation, obstruction, fistula, or abscess formation are suspected $[17,18]$.

Particularly in patients with penetrating $\mathrm{CD}$, the anterior abdominal wall should be carefully scrutinised on crosssectional imaging studies. Sometimes unexpected or subtle, ECFs are recognised at CT as "tram-track" structures with fluid and/or gaseous content (Fig. 8), which generally depart from the site of active disease, entero-enteric fistulas, or anastomotic $\mathrm{CD}$ recurrence. With MRI, fluid-filled ECFs are well recognised along their course from the bowel to the skin (Fig. 9). In the setting of active IBD, a characteristic appearance is the mucosal contrast enhancement, which is generally prominent and appreciated on either CT (Fig. 8) and on fat-suppressed MRI sequences (Figs. 9 and 10). More common than with post-surgical ECFs, abscess incision, from the apex of a large T2-hyperintense mass (* in A) corresponding to recurrent ASC. Post-gadolinium fat-suppressed T1-weighted image (C) showed mucosal hyperenhancement (thin arrow) along the fistula track

formation is characteristic of CD: with both modalities, abscess collections with peripheral enhancement, and mixed air, fluid and dense content are easily detected at either internal opening or along the subcutaneous track (Fig. 10) [19, 20].

\section{Post-surgical enterocutaneous fistulas in CD}

Alternatively, an ECF may occur within a few weeks or months after IBD surgery, often through a previous abdominal scar and without residual or recurrent disease; clinically, this situation is commonly associated with limited physical findings due to use of steroids or immunomodulators.

In patients who received surgery recently, multidetector CT (Fig. 11A-C) is preferred to confirm and visualise ECFs, with similar imaging findings to their spontaneous counterparts. Unfortunately, interpretation of cross-sectional imaging studies shortly after IBD surgery is generally difficult due to a combination of limited intra-abdominal fat and of postoperative changes such as diffuse oedema at resection site and in the mesentery, peritoneal fluid, and CM enhancement; in our experience this is particularly true with MRI (Fig. 11D-F) [15, 16].

\section{Treatment}

The optimal treatment of enterocutaneous fistulas in CD remains debated: in the past, surgical resection of the diseased bowel plus fistula debridement through abdominal wall and subcutaneous planes was generally performed. In the last decade, medical therapy changed with introduction of biologics and immunomodulators. Following experience with perianal inflammatory disease, monoclonal antibodies against tumour necrosis factor are increasingly used: with correct patient 
Fig. 8 Two cases of spontaneous, low-output ECF in CD. In a 59year-old man with a history of ileocecal resection nearly 20 years earlier, CT (A, B) identified a right-sided ECF as an enhancing "tram-track" fistula (arrows) with mucosal hyperenhancement (thin arrow), which departed from the thickened preanastomotic ileum (thick arrow) with recurrent CD and crossed the abdominal wall and subcutaneous fat to the EFO (arrowheads); repeated ileocolonic resection and ECF debridement were performed In a 62-year-old woman with several surgeries, water-enema CT (C, D - note distended rectosigmoid colon with rectal tube) showed a paramedian hypogastric ECF with mucosal hyperenhancement (thin arrow) and focally depressed EFO (arrowheads), communicating with the collapsed ileum; the patient did well on infliximab therapy
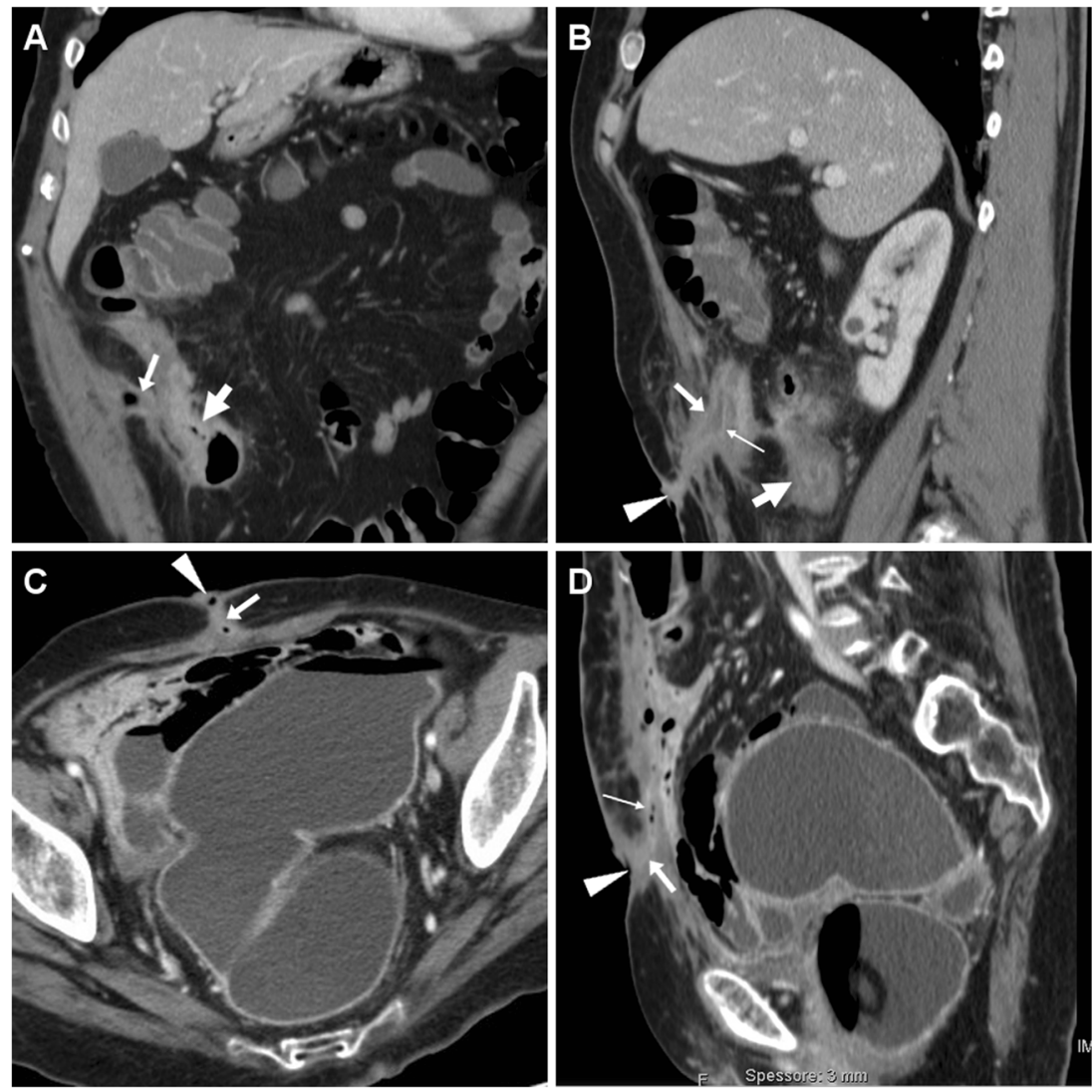

selection, in the absence of stenosis and complex fistulas, infliximab achieves good response in postoperative and, to a minor extent, spontaneous ECFs too [16, 21, 22].

\section{Special situation: malignant fistulas in Crohn's disease}

In patients with long-standing $\mathrm{CD}$, small bowel adenocarcinoma (SBAC) occurs 33 times more commonly than in the general population. Unfortunately, its unspecific manifestations mimic those of active or obstructive $\mathrm{CD}$, resulting in frequently delayed diagnosis at an advanced stage with disseminated disease $[23,24]$.

Sometimes, SBAC may penetrate through the anterior abdominal wall and fistulise to the skin (Fig. 12), a situation that is easily misinterpreted as the usual, spontaneous CDrelated ECF unless thorough imaging is obtained.

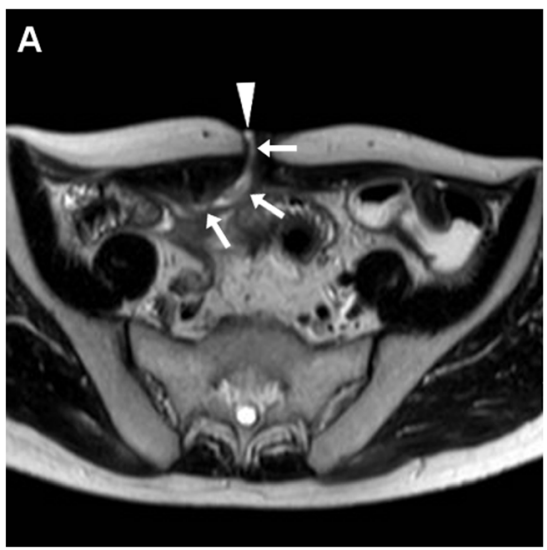

Fig. 9 Spontaneous ECF in a 37-year-old man with stricturing and fistulising $\mathrm{CD}$, shown at MR-enterography as a fluid-like T2-hyperintense track (arrows in A) coursing from an ileal loop through the abdominal wall, and finally reaching the EFO (arrowheads). Post-gadolinium

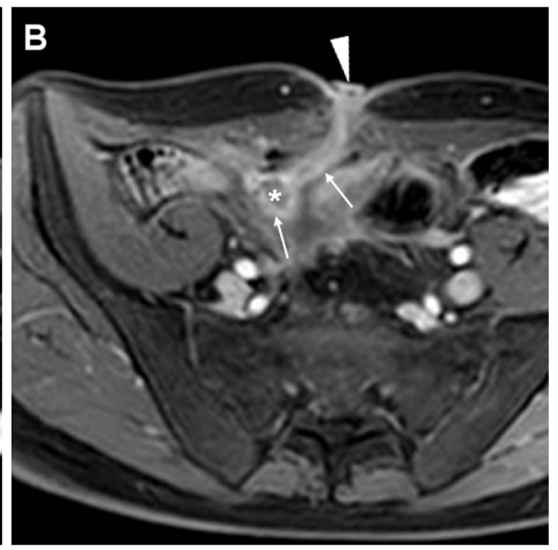

fat-suppressed T1-weighted acquisition (B) showed mucosal hyperenhancement along ECF track (thin arrows) and at site of internal opening (*). Surgical treatment required fistula debridement plus stricturoplasty 

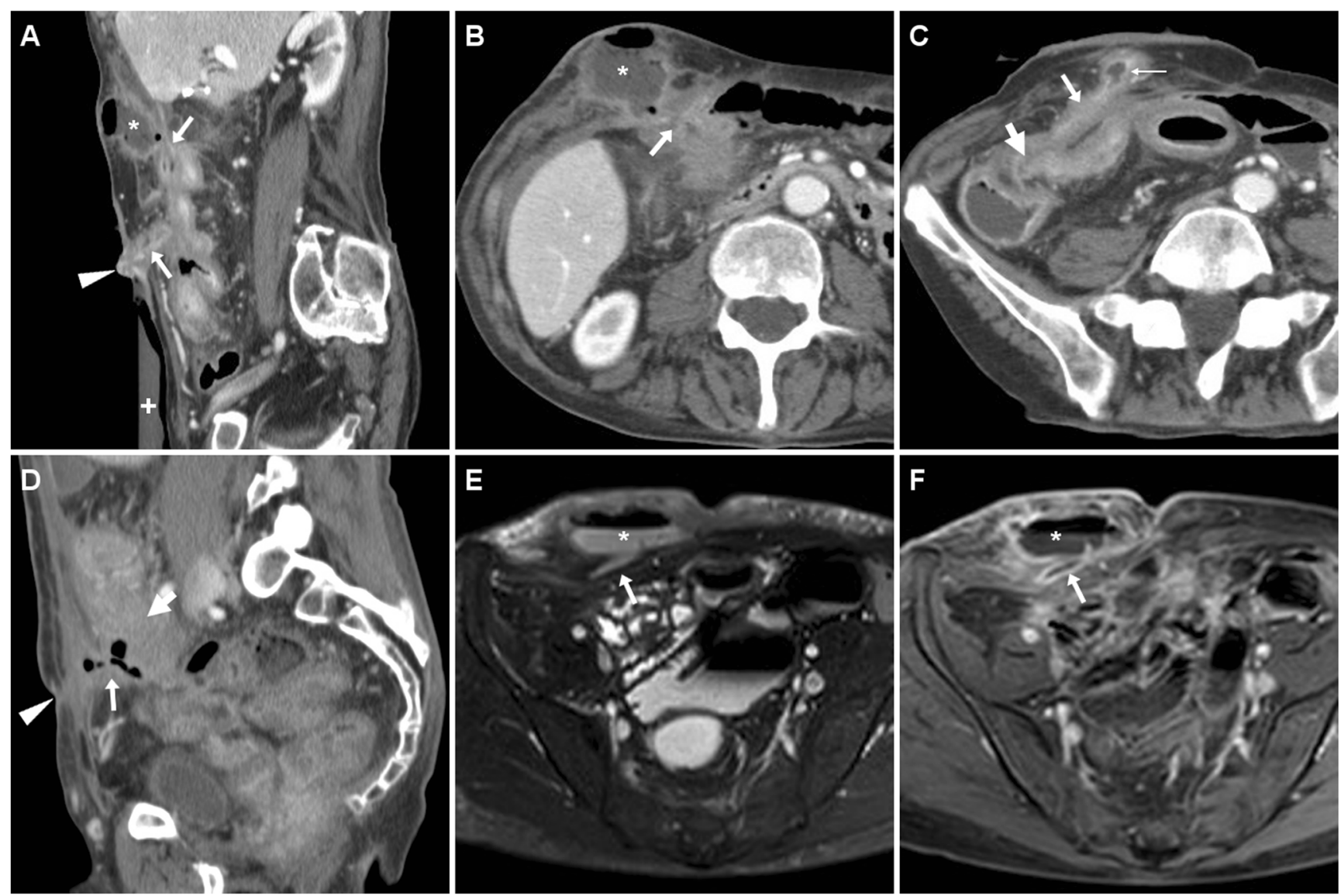

Fig. 10 Two cases of spontaneous ECF complicated by abscess collections in chronic inflammatory bowel diseases. A 77-year-old man with indeterminate colitis treated by proctocolectomy and definitive ileostomy developed two ECFs, one in right hypochondrium with a sizeable superficial abscess ( $*$ in A, B) communicating (arrows) with a jejunal loop; the second, longer and more distal ECF (arrow in $\mathbf{C}$ ) originated from the thickened ileum (thick arrow) and included a smaller subcutaneous abscess with inflamed mucosa (thin arrow). In a 59-year-old woman with fistulising CD, the ECF corresponded at CT (D) to a gasfilled track (arrow) arising from the diseased ileocolonic anastomosis (thick arrow) and reaching a subtle depressed EFO (arrowhead). Follow-up MRI including T2- (E) and post-contrast fat-saturated T1weighted (F) showed development of a large abscess (*) with fluid content and enhancing wall along the ECF (arrows); definitive surgical treatment required extensive adhesiolysis, resection of rectum and of diseased perianastomotic bowel
Unfortunately, differentiation of SBAC from either acute inflammatory or fibrotic $\mathrm{CD}$ is challenging. The commonest cross-sectional imaging patterns include ileal mass, long stricture with heterogeneous submucosa, short severe stenosis with upstream bowel dilatation, irregular asymmetric circumferential thickening. Suspicious features include mural thickness $>1 \mathrm{~cm}$, abrupt "shouldering" transition, lost mural stratification, soft-tissue attenuation or solid MRI signal intensity, irregular serosal nodularity, and adenopathies (Fig. 12) [25-27].

The rare possibility of SBAC should be considered in patients with worsening CD symptoms after long quiescence and in non-healing ECFs despite treatment. Albeit difficult, preoperative diagnosis of malignant ECF in CD impacts treatment since it requires en bloc resection plus chemotherapy $[23,24]$. Alternatively, the development of squamous cell carcinoma in chronic CD-related ECF has been occasionally reported [28].

\section{Irradiation}

Albeit most patients receiving abdominal or pelvic irradiation develop some degree of radiation enteritis, post-radiation ECF are increasingly rare and develop at least 4-6 months after radiotherapy. Unfortunately, irradiation further increases complexity of management, due to a combination of three factors: a) low likelihood of spontaneous closure; b) poor healing and high risk of dehiscence following surgery on irradiated bowel; c) delayed or impossible adjuvant or palliative therapies in presence of an ECF [4].

\section{Tumour-related enterocutaneous fistulas}

The possibility of an underlying malignancy should be considered when faced with a spontaneous ECF in an adult or elderly patient without history of recent surgery 

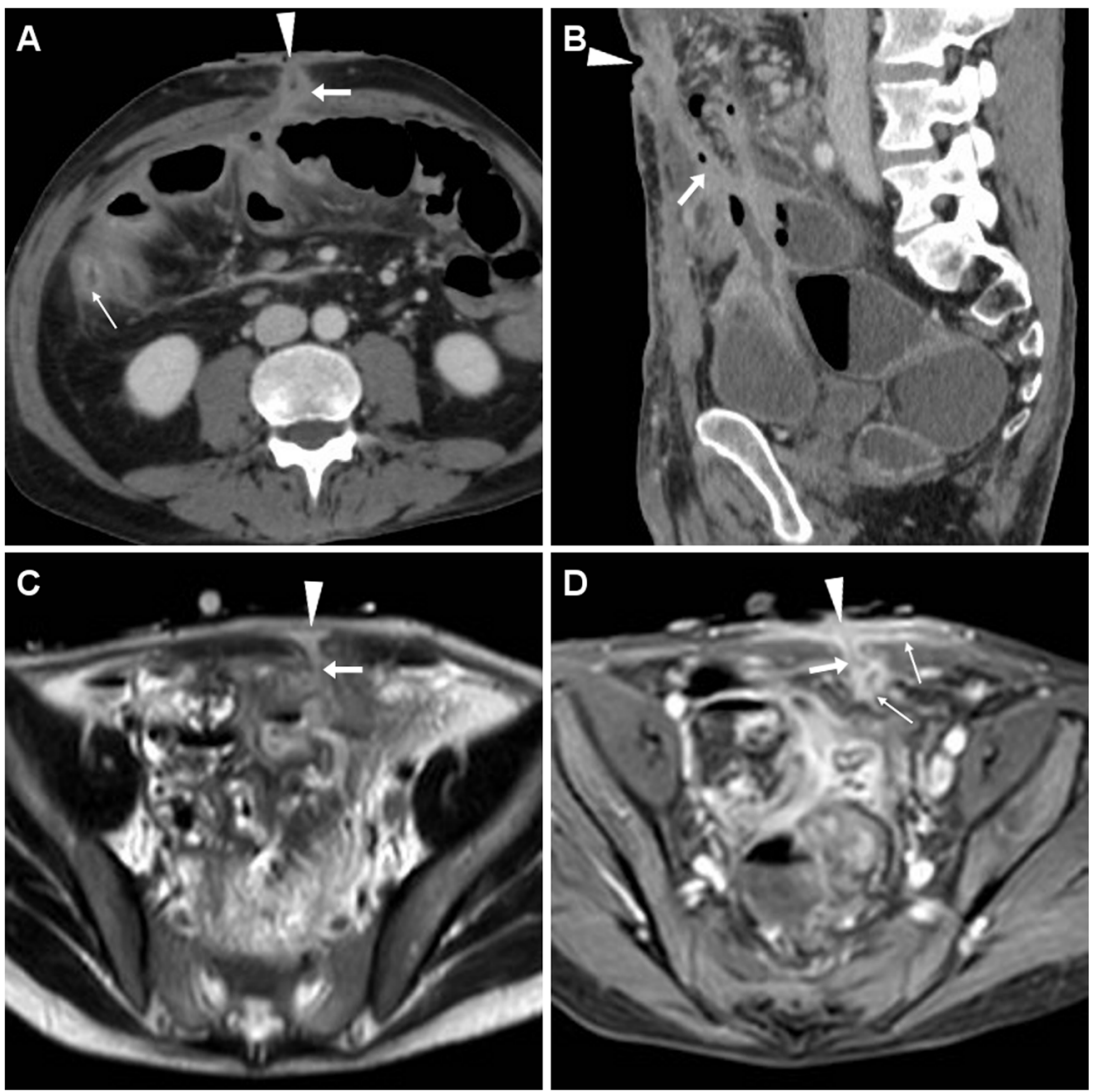

Fig. 11 Two cases of post-surgical ECF in CD. After recent ileocecal resection, in a 61-year-old man multiplanar CT-enterography $(\mathbf{A}, \mathbf{B})$ showed mural thickening with mucosal hyperenhancement (thin arrows) along distal ileum consistent with disease activity, and an ECF (arrows) coursing obliquely from the affected tract to small-sized mesogastric EFO (arrowheads); reoperation included ECF debridement, ileal resection and redo anastomosis. A 27-year-old man developed an ECF after subtotal

or IBD. This situation is increasingly rare since most colorectal malignancies are currently diagnosed and treated at an early or preclinical stage, but are strongly
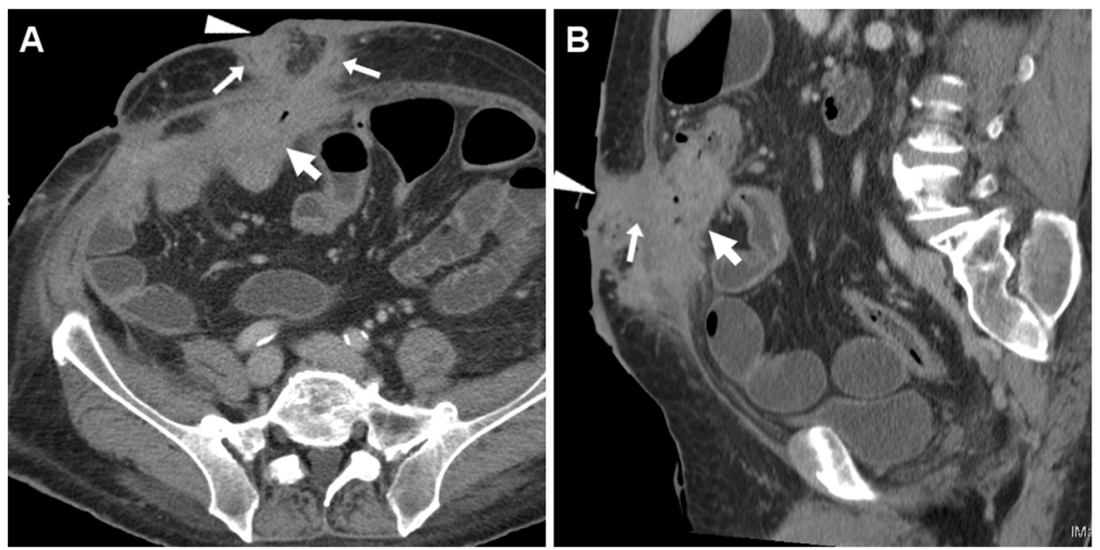

Fig. 12 Ileal adenocarcinoma fistulising to the skin in a 68-year-old man with long-standing $\mathrm{CD}$, previous ileocecal resection, recent weight loss and two draining EFOs at the anterior abdominal wall. CT-enterography confirmed double ventral ECF (arrows) from a markedly thickened nonstratified ileal segment (thick arrows). Neoplastic infiltration of the

colectomy with cecal-rectum anastomosis, with corresponding MRI (T2weighted image $\mathbf{C}$, fat-suppressed post-contrast T1-weighted image D) appearance of fluid-filled track (arrows) with peripheral enhancement (thin arrows). Prominent inflammation also affected the skin surrounding the EFO (arrowheads) and at inner aspect of the ECF: the latter corresponded endoscopically to the anastomotic site and was treated by positioning of an over-the-scope clip, allowing ECF resolution

associated (over $50 \%$ of cases) with disseminated disease, impaired quality of life and high 30-day mortality $[29,30]$.

anterior abdominal wall was pathologically confirmed after en-bloc surgical resection. Tumour progression with ascites and liver metastases (not shown) ultimately developed (Partially reproduced from Open Access ref. no [34]) 


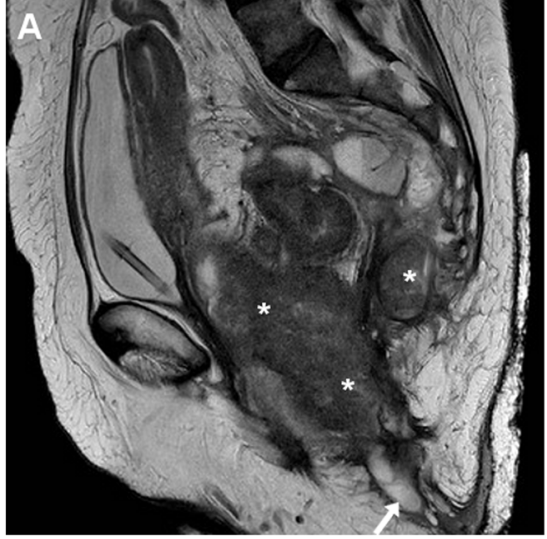

Fig. 13 Locally advanced rectal cancer with superinfection and ECF formation in a 54-year-old woman with septic fever. T2-weighted MRI images (A) showed a large solid tumour (*) invading the vagina and perineum. Additionally, a 1-cm wide fluid-containing ECF (arrows)

Tumour-related ECFs result from either superinfection (Fig. 13) or fistulisation through the abdominal wall muscles (Fig. 14). In both cases, CT and MRI are crucial in identifying solid, more or less homogeneous and enhancing neoplastic masses (Fig. 13) and the possible presence of abscess collections requiring drainage. Alternatively, cross-sectional imaging suggests ECFassociated colorectal tumour when faced with irregular or asymmetric mural thickening (Fig. 14); features such as short segmental involvement, abrupt transition, lost mural stratification and lymphadenopathy further support a diagnosis of malignancy [31-33].

The ideal curative surgery is en-bloc removal of fistulising tumour along with the involved abdominal wall along the ECF track, plus derivative colostomy or

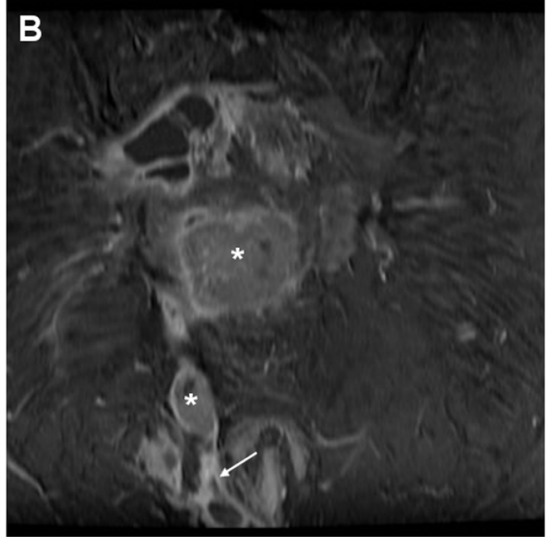

coursed postero-inferiorly towards the perianal skin and showed peripheral enhancement (thin arrow) on post-gadolinium fat-suppressed T1weighted acquisition (B)

ileostomy. Alternative options include palliative resection, bypass or stoma in unresectable tumours $[10,30]$.

\section{Conclusion}

Nowadays, most patients with ECFs undergo multidetector $\mathrm{CT}$ or MRI studies to provide comprehensive cross-sectional assessment as a consistent basis for appropriate treatment choice and planning. In fact, visualisation of the underlying conditions (such as postoperative anastomotic leak, bowel obstruction, abscesses amenable to drainage, active or stricturing $\mathrm{CD}$, fistulising or recurrent tumour) allows directed treatment, thus resulting in decreased rates of failed conservative management and higher likelihood of therapeutic success [4, 5].
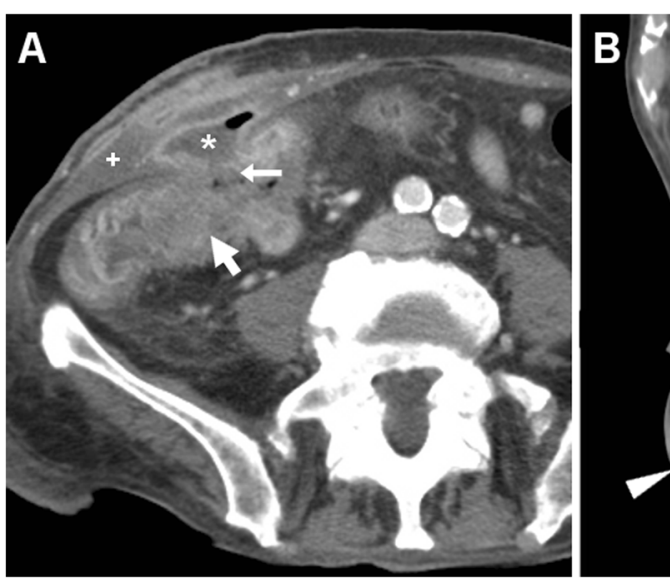

Fig. 14 Cutaneous fistulisation of colon carcinoma in a 92-year-old man with worsening right-sided abdominal pain and tenderness. CT detected a segmental, non-stratified circumferential thickening (thick arrows) at the ascending colon, which infiltrated the peritoneum, opened (arrows in A, B) into an abscess $\left(^{*}\right)$ in the inflamed abdominal wall (+), and

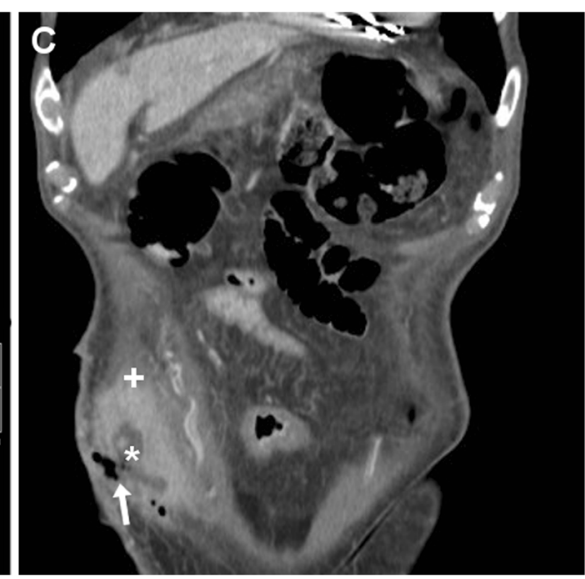

communicated to the skin EFO (arrowhead in B) through an ECF (arrow in C). Liver metastases (thick arrow) were present. The patient was treated supportively with positioning of colostomy-type bag at the EFO (Partially reproduced from Open Access ref. no [35]) 
Open Access This article is distributed under the terms of the Creative Commons Attribution 4.0 International License (http:// creativecommons.org/licenses/by/4.0/), which permits unrestricted use, distribution, and reproduction in any medium, provided you give appropriate credit to the original author(s) and the source, provide a link to the Creative Commons license, and indicate if changes were made.

\section{References}

1. Murphy J, Hotouras A, Koers L et al (2013) Establishing a regional enterocutaneous fistula service: the Royal London hospital experience. Int J Surg 11:952-956

2. Orangio GR (2010) Enterocutaneous fistula: medical and surgical management including patients with Crohn's disease. Clin Colon Rectal Surg 23:169-175

3. Visschers RG, van Gemert WG, Winkens B et al (2012) Guided treatment improves outcome of patients with enterocutaneous fistulas. World J Surg 36:2341-2348

4. Felipe de Campos-Lobato L, Vogel JD (2010) Enterocutaneous fistula associated with malignancy and prior radiation therapy. Clin Colon Rectal Surg 23:176-181

5. Lee JK, Stein SL (2010) Radiographic and endoscopic diagnosis and treatment of enterocutaneous fistulas. Clin Colon Rectal Surg 23:149-160

6. Gyorki DE, Brooks CE, Gett R et al (2010) Enterocutaneous fistula: a single-centre experience. ANZ J Surg 80:178-181

7. Williams LJ, Zolfaghari S, Boushey RP (2010) Complications of enterocutaneous fistulas and their management. Clin Colon Rectal Surg 23:209-220

8. Gu GL, Wang L, Wei XM et al (2014) Necrotizing fasciitis secondary to enterocutaneous fistula: three case reports. World $\mathrm{J}$ Gastroenterol 20:7988-7992

9. Redden MH, Ramsay P, Humphries T et al (2013) The etiology of enterocutaneous fistula predicts outcome. Ochsner J 13:507-511

10. Chamberlain RS, Kaufman HL, Danforth DN (1998) Enterocutaneous fistula in cancer patients: etiology, management, outcome, and impact on further treatment. Am Surg 64:1204-1211

11. Foda M, Carlson MA (2009) Enterocutaneous fistula associated with ePTFE mesh: case report and review of the literature. Hernia 13:323-326

12. Tonolini M, Ippolito S (2016) Multidetector CT of expected findings and early postoperative complications after current techniques for ventral hernia repair. Insights Imaging 7:541-551

13. Mauri G, Pescatori LC, Mattiuz C et al (2017) Non-healing postsurgical fistulae: treatment with image-guided percutaneous injection of cyanoacrylic glue. Radiol Med 122:88-94

14. Lee SH (2012) Surgical management of enterocutaneous fistula. Korean J Radiol 13(Suppl 1):S17-S20

15. Cellini C, Safar B, Fleshman J (2010) Surgical management of pyogenic complications of Crohn's disease. Inflamm Bowel Dis 16:512-517

16. Gomez-Senent S, Barreiro-de-Acosta M, Garcia-Sanchez V (2013) Enterocutaneous fistulas and Crohn s disease: clinical characteristics and response to treatment. Rev Esp Enferm Dig 105:3-6

17. Panes J, Bouhnik Y, Reinisch W et al (2013) Imaging techniques for assessment of inflammatory bowel disease: joint ECCO and
ESGAR evidence-based consensus guidelines. J Crohns Colitis 7: $556-585$

18. Norsa AH, Tonolini M, Ippolito S et al (2013) Water enema multidetector $\mathrm{CT}$ technique and imaging of diverticulitis and chronic inflammatory bowel diseases. Insights Imaging 4:309-320

19. Griffin N, Grant LA, Anderson S et al (2012) Small bowel MR enterography: problem solving in Crohn's disease. Insights Imaging 3:251-263

20. Baker ME, Hara AK, Platt JF et al (2015) CT enterography for Crohn's disease: optimal technique and imaging issues. Abdom Imaging 40:938-952

21. Fries W, La Malfa G, Costantino G et al (2011) Combined approach with biologics and surgery for enterocutaneous fistulas in Crohn's disease. Inflamm Bowel Dis 17:671-673

22. Amiot A, Setakhr V, Seksik P et al (2014) Long-term outcome of enterocutaneous fistula in patients with Crohn's disease treated with anti-TNF therapy: a cohort study from the GETAID. Am J Gastroenterol 109:1443-1449

23. Elriz K, Carrat F, Carbonnel F et al (2013) Incidence, presentation, and prognosis of small bowel adenocarcinoma in patients with small bowel Crohn's disease: a prospective observational study. Inflamm Bowel Dis 19:1823-1826

24. Widmar M, Greenstein AJ, Sachar DB et al (2011) Small bowel adenocarcinoma in Crohn's disease. J Gastrointest Surg 15:797802

25. Place V, Hristova L, Dray X et al (2012) Ileal adenocarcinoma in Crohn's disease: magnetic resonance enterography features. Clin Imaging 36:24-28

26. Soyer P, Hristova L, Boudghene F et al (2012) Small bowel adenocarcinoma in Crohn disease: CT-enterography features with pathological correlation. Abdom Imaging 37:338-349

27. Weber NK, Fletcher JG, Fidler JL et al (2015) Clinical characteristics and imaging features of small bowel adenocarcinomas in Crohn's disease. Abdom Imaging 40:1060-1067

28. Pinto Pais T, Fernandes S, Carvalho J (2014) Squamous cell carcinoma in enterocutaneous fistula associated with Crohn's disease: first case report. J Crohns Colitis 8:1142-1143

29. Gunay Y, Bircan H, Demiralay E et al (2013) Spontaneous enterocutaneous fistula: unusual presentation of colon cancer. Turk J Gastroenterol 24:458-459

30. Osian G (2012) Emergency Surgery for Colorectal Cancer Complications: Obstruction, Perforation, Bleeding. Contemporary Issues in Colorectal Surgical Practice, Dr Yik-Hong Ho (Ed) InTech, Available from: http://www.intechopen.com/books/ contemporary-issues-in-colorectal-surgicalpractice/emergencysurgery-for-colorectal-cancer-complications

31. Catalano O (1997) Perforated carcinoma of the colorectum. Computerized tomography findings. Radiol Med 93:83-86

32. Kim SW, Shin HC, Kim IY et al (2010) CT findings of colonic complications associated with colon cancer. Korean J Radiol 11: 211-221

33. Tonolini M, Bianco R (2013) MRI and CT of anal carcinoma: a pictorial review. Insights Imaging 4:53-62

34. Tonolini M, Sampietro G (2016) Malignant enterocutaneous fistula in Crohn's disease \{Online\}. EuroRAD http://www.eurorad.org/ case.php?id=13194

35. Tonolini M (2016) Spontaneous cutaneous fistulisation of colon carcinoma \{Online\}. EuroRAD http://www.eurorad.org/case.php? id $=13353$ 